DIMACS Series in Discrete Mathematic

and Theoretical Computer Science

Volume on, $19 \times \mathrm{x}$

\title{
A Branch and Cut Algorithm for MAX-SAT and Weighted MAX-SAT
}

\author{
Steve Joy, John Mitchell, and Brian Borchers
}

\begin{abstract}
We describe a branch and cut algorithm for both MAX-SAT and weighted MAX-SAT. This algorithm uses the GSAT procedure as a primal heuristic. At each node we solve a linear programming (LP) relaxation of the problem. Two styles of separating cuts are added: resolution cuts and odd cycle inequalities.

We compare our algorithm to an extension of the Davis Putnam Loveland (EDPL) algorithm. Our algorithm is more effective than EDPL on some problems, notably MAX-2-SAT. EDPL is more effective on some other classes of problems.
\end{abstract}

\section{Introduction}

The satisfiability problem (SAT) is a problem in propositional logic. A logic formula consists of the conjunction of clauses. Each clause consists of a disjunction of literals. Each literal is a variable or its negation. The SAT problem seeks to find an assignment to the variables which satisfies the logic formula, or an indication that no such assignment exists. The satisfiability problem is NP-complete [5].

There are a number of exact algorithms for the satisfiability problem. These include Davis-Putnam-Loveland $[\mathbf{4}, \mathbf{2 3}]$, resolution [26], and integer programming approaches $[\mathbf{1}, \mathbf{1 6}, \mathbf{1 8}, \mathbf{2 0}, \mathbf{2 1}]$. A number of heuristics that use randomization also exist; the first randomized local search algorithm for satisfiability was due to Gu $[\mathbf{9}, \mathbf{1 0}, \mathbf{1 1}, \mathbf{1 2}]$. Other algorithms include the GSAT heuristic $[\mathbf{2 9}, \mathbf{3 0}]$ and the GRASP heuristic [25]. For surveys of algorithms for SAT problems see [13, 14].

In this paper we investigate the related MAX-SAT problem. Given a collection of clauses, we seek a variable assignment that maximizes the number of satisfied clauses. The weighted MAX-SAT problem assigns a weight to each clause, and seeks an assignment that maximizes the sum of the weights of the satisfied clauses. Both of these problems are NP-hard. It is possible to approximate MAX-SAT within a factor of 1.325 in polynomial time [6].

Most SAT heuristics have been extended to MAX-SAT. Several heuristics for MAX-SAT are summarized in Hansen and Jaumard [15]. The GSAT heuristic has also been extended to weighted MAX-SAT [22].

1991 Mathematics Subject Classification. 03B05, 49M35, 65K05, 90C10.

Research supported by ONR Grant N00014-94-1-0391 to Rensselaer Polytechnic Institute.

(C)000 American Mathematical Society $1052-1798 / 00 \$ 1.00+\$ 25$ per page 
In this paper we investigate a branch and cut approach to MAX-SAT. We then report on computational results using both our approach and an extension of the Davis-Putnam-Loveland procedure [2].

1.1. MAX-SAT as an Integer Programming Problem. A logical variable $v_{i}$ can be TRUE or FALSE. We replace this variable with a corresponding integer variable $x_{i}$. This variable takes on value 1 when $v_{i}$ is TRUE and 0 when it is FALSE.

An unnegated literal $v_{i}$ is simply replaced with the expression $x_{i}$. A negated literal such as $\overline{v_{i}}$ can be replaced with the expression $1-x_{i}$. When $v_{i}$ is TRUE, $\overline{v_{i}}$ is FALSE; $x_{i}$ is 1 , and $1-x_{i}$ is 0 . When $v_{i}$ is FALSE, $\overline{v_{i}}$ is TRUE; $x_{i}$ is 0 , and $1-x_{i}$ is 1 .

A clause is satisfied if, and only if, at least one of its $k$ literals is TRUE. For the integer problem, we sum the corresponding $\mathrm{k}$ expressions. The clause is true if, and only if, the sum is one or more. For example, the clause

$$
\overline{v_{1}} \vee v_{3} \vee v_{7} \vee \overline{v_{9}}
$$

is equivalent to

$$
\left(1-x_{1}\right)+x_{3}+x_{7}+\left(1-x_{9}\right) \geq 1
$$

We must have some way of handling the maximization of satisfied constraints. We do this by adding variables to the problem. A clause is either satisfied or it isn't. But this just produces a new clause that is always satisfied:

\section{original clause $\vee$ original clause not satisfied}

we can replace the 2 nd term above with a new variable. There will be one such variable per clause.

Maximizing the sum of the weights of satisfied constraints is equivalent to minimizing the sum of the weights of unsatisfied constraints. This sum is simply the sum of the weight of each clause times the variable indicating that the clause is not satisfied. Given the MAX-SAT problem:

$$
\begin{array}{ll}
\overline{v_{1}} \vee \overline{v_{2}} & \text { weight } 1 \\
v_{1} \vee v_{2} \vee v_{3} & \text { weight } 4 \\
v_{1} \vee \overline{v_{2}} & \text { weight } 3
\end{array}
$$

we obtain the equivalent Integer Program (IP):

$$
\begin{aligned}
\min & w_{1}+4 w_{2}+3 w_{3} \\
\text { s.t. } \quad\left(1-x_{1}\right) & +\left(1-x_{2}\right) \\
x_{1}+x_{2}+x_{3} & +w_{1} \\
x_{1}+\left(1-x_{2}\right) & +w_{2} \\
& +w_{3} \geq 1 \\
x_{i}=0 \text { or } 1, i=1, \ldots, 3 . w_{i}=0 \text { or } 1, i=1, \ldots, 3 &
\end{aligned}
$$

or 


$$
\begin{gathered}
\text { min } \\
\text { s.t. }-w_{1}+4 w_{2}+3 w_{3} \\
\\
\end{gathered}
$$

$x_{i}=0$ or $1, i=1, \ldots, 3 . w_{i}=0$ or $1, i=1, \ldots, 3$

These added variables are referred to as the weighted variables. The other variables are the unweighted variables.

\section{Description of Algorithm}

2.1. Overview. The basic approach is branch and cut $[7, \mathbf{8}, \mathbf{1 7}, \mathbf{2 4}]$. At each node of the tree we solve the linear programming (LP) relaxation obtained by replacing the integrality requirements by the simple bounds:

$0 \leq x_{i} \leq 1, i=1, \ldots, n, 0 \leq w_{i} \leq 1, i=1, \ldots, m$

If the solution to the LP is integral, we compare it to the best integral solution so far. If the solution has one or more fractional variables, we branch on one of the fractional variables and repeat the procedure.

The algorithm has several interacting components. A primal heuristic is used to obtain an upper bound on the binary solution. This allows us to fathom nodes in the branch and cut tree. A bounds routine is used to determine if any variable of the LP relaxation can be fixed at zero or one. Resolution cuts and odd cycle inequalities are added to guide the LP toward a binary solution. Finally, a branching routine is used to choose a variable on which to branch.

2.2. Primal Heuristic. The primal heuristic is run once at the beginning of the algorithm. This routine is an efficient randomized local search heuristic, similar to other good heuristics in the literature $[\mathbf{9}, \mathbf{1 0}, \mathbf{1 1}, \mathbf{1 2}, \mathbf{2 2}, \mathbf{2 9}, \mathbf{3 0}]$. Several tries are attempted. For each try we randomly choose a binary assignment to the variables. We then perform a series of flips. By flipping variables (choosing the opposite assignment for a single variable), we attempt to move towards an optimal solution.

There are two types of flips. Random flips randomly select a variable to flip. Best-choice flips attempt to select the best variable to flip. We compute the effect of a flip, that is the net increase or decrease in the sum of the weights of the satisfied clauses. We choose the variable with the best net increase in this sum. If several variables produce the same net increase, we select one of these variables at random.

Seventy percent of the flips (chosen arbitrarily) are best-choice flips. The random flips are used to assist in escaping from local minima.

2.3. Node Fathoming. The difference between the optimal value of the LP relaxation and that of the incumbent solution is referred to as the gap. If the gap is sufficiently small then we can fathom the node. That is, we know that any binary solution along this branch is no better than the incumbent solution.

If the gap is strictly less than some threshold then we can fathom the nodes. Normally this threshold is the greatest common divisor of the clause weights. In the special case where the optimal incumbent solution has one unsatisfied clause of minimal weight, the threshold is this minimum weight. 
2.4. Variable bounds. In some cases we can fix variables, thus simplifying the problem. When a variable is fixed, some clauses become satisfied.

Let us sum the weighted variables that are fixed at one. This is the fixed weighted variable sum, and it represents a lower bound on the LP objective function. Suppose we have a non-satisfied clause whose weight when added to this sum is greater than or equal to the optimal value of the incumbent solution. Since we are seeking a better solution, the weighted variable of the clause must be fixed at zero (or equivalently the unweighted portion of the clause must be satisfied). This clause is a must satisfy clause.

A simple form of variable fixing is sufficient weight fixing. If we have a must satisfy clause then we fix the weighted variable at zero.

If we have a must satisfy clause with only a single unfixed unweighted variable then we must fix this variable in such a way as to satisfy the clause. This is known as unit clause fixing.

If a variable appears in only the positive sense in the non-satisfied clauses then we can fix the variable at one. Likewise, if the variable is always negated then we can fix it at zero. This is known as monotone variable fixing.

First, we examine the clauses that are not satisfied. We compute the number of non-fixed variables in each clause. For each non-fixed variable, we count the number of clauses in which it appears in the positive and negated senses. If a variable appears in only the positive sense then fix it via monotone variable fixing. If there is only a single non-fixed variable in a clause of sufficient weight then fix it via unit clause fixing.

When a variable is fixed, this satisfies some constraints. We decrement the counters for the other variables in this newly satisfied constraint. This potentially allows us to fix additional variables that may now have become monotone. As we fix a variable, all constraints having the opposite sense of the variable are now shorter. If a clause is now of length one and has sufficient weight then we can fix the remaining variable via unit clause fixing.

If an original constraint is satisfied, we set the clause's weighted variable to zero. This is known as satisfied clause fixing.

If all the unweighted variables of an original clause are fixed in such a way that none of the unweighted literals satisfy the clause then we set the clause's weighted variable to one. This is known as unsatisfied clause fixing.

2.5. Cut generation. There are two types of cuts: resolution cuts and odd cycle inequalities. The cuts are applied locally (at this node of the branch and bound tree and its descendents) rather than to the whole tree. Resolution cuts are discussed by Hooker and Fedjki $[\mathbf{1 8}, \mathbf{1 9}, \mathbf{2 0}]$. Odd cycle inequalities are discussed by Cheriyan et al. [3].

2.5.1. Resolution cuts. Resolution cuts arise by combining two clauses: one with the positive sense of a variable, the other clause with the negative sense. There must be exactly one such literal. The resolvent consists of literals (even those arising from weighted variables) found in either clause except for the variable of opposite sense. For example, given the clauses:

$$
\begin{aligned}
& \overline{x_{1}} \vee x_{3} \vee x_{7} \vee w_{9} \\
& x_{2} \vee \overline{x_{3}} \vee x_{7} \vee w_{11}
\end{aligned}
$$


we can resolve to generate the following:

$$
\overline{x_{1}} \vee x_{2} \vee x_{7} \vee w_{9} \vee w_{11}
$$

The resolution routine is activated once at each node of the tree. It consists of a sequence of passes, each designed to generate resolvents matching certain criteria. In each pass for each variable of each resolvable clause, the list of clauses with the opposite sense of that variable is obtained. This clause is resolved (if possible) with each of the other clauses on the list. If the resolvent matches the given criteria, it is added to the list of resolvable clauses for the next pass. If this clause is of length one or less (counting only the original unweighted variables) and is a sufficiently deep separating cut (violated by 0.3 or more with the current LP solution) then it is added to the list of separating cuts (the length and depth restrictions were determined by experimentation to yield the fastest algorithms). This process continues until a sufficient number of resolvents have been generated or all such clauses have been exhausted.

At the end of each pass, constraints that can not possibly be useful in future passes are removed from consideration. The resolvents that are generated are reduced to a minimal set through absorption. In absorption if the unweighted literals of clause A are a subset of the literals of clause B, then clause A implies clause B. In this case, clause B is absorbed by clause A and B is removed from consideration. The list of separating cuts is also reduced to a minimal set through absorption.

The first pass requires that the resolvents match the requirements for the separating cuts, that is, of length one or less and violated by 0.3 or more.

The second pass requires that the resolvents be of length three or less.

The third pass requires that the resolvents be of length two or less.

The fourth pass requires that the resolvents be of length one or less.

2.5.2. Odd cycle inequalities. The odd cycle inequalities combine clauses with two unfixed unweighted variables. A clause is considered odd if both unfixed unweighted variables are negated, or if both are not negated; otherwise, the clause is considered even. A cycle $x_{i_{1}}, \ldots, x_{i_{k}}$ in the unfixed unweighted variables is sought. The first constraint involves variables $x_{i_{1}}$ and $x_{i_{2}}$, the next $x_{i_{2}}$ and $x_{i_{3}}$, the next $x_{i_{3}}$ and $x_{i_{4}}, \ldots$ the last clause involving $x_{i_{k}}$ and $x_{i_{1}}$. The cycle is said to be odd if when we "add up" these constraints, we obtain an odd total. By insuring that the cycle is odd, we know that the right hand side will be odd, and will be rounded up when we divide the coefficients of the resulting constraint by two.

This is best illustrated by an example. Suppose we have the constraints below. Variables $x_{1}-x_{4}$ are unfixed unweighted variables, the other variables are unfixed and weighted. The first constraint is a cut (generated at a previous branch and cut node) since it involves multiple weighted variables.

$$
\begin{aligned}
& \begin{array}{llll}
x_{1}+x_{2} & +w_{10}+w_{11} & \geq 1
\end{array}
\end{aligned}
$$

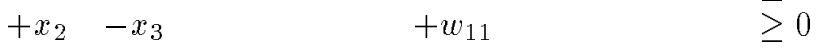

$$
\begin{aligned}
& \begin{array}{rlll}
-x_{3} & -x_{4} & +w_{12} & \geq-1 \\
& -x_{4} & & \geq w_{13}
\end{array} \\
& \begin{array}{lll}
-x_{1} & -x_{4} & +w_{13} \geq-1
\end{array}
\end{aligned}
$$

The first, third, and fourth constraints are odd; the second is even. Adding these constraints together we obtain:

$$
2 x_{2}-2 x_{3}-2 x_{4}+w_{10}+2 w_{11}+w_{12}+w_{13} \geq-1
$$


Dividing by 2 and rounding coefficients we obtain:

$$
x_{2}-x_{3}-x_{4}+w_{10}+w_{11}+w_{12}+w_{13} \geq 0
$$

We are again seeking only separating cuts. Odd cycles force this increase in the right hand side thus potentially generating a separating cut. Even cycles do not have this potential. If we find an odd cycle of accumulated weight (defined below) $1-2 \delta$, then we obtain a separating cut of depth $\delta$. We are seeking a cut of depth 0.3 , so we are seeking an odd cycle of weight 0.4 or less. We also require that the cut is of length two or less (the length and depth restrictions were determined by experimentation to yield the fastest algorithms).

We use a modified version of Dijkstra's shortest path algorithm to find the separating cuts. Dijkstra's algorithm starts from a given start node of a tree and uses edge weights to find the shortest path from this start node to any other. Initially, the start node is assigned weight 0 . All others are assigned infinite weight. All nodes are initially unmarked. The algorithm then picks the lowest weight unmarked node. This node is now marked. For any unmarked neighbors of this chosen node with

chosen weight + weight of edge from chosen to neighbor < weight of neighbor

we replace the neighbor's weight with this sum. This process continues until all the shortest paths have been found (all nodes are marked). The algorithm can be slightly modified to record the actual shortest path to a node (in addition to its length).

We use the algorithm once per variable. In each iteration this variable is the start variable.

- The edges represent clauses with two unfixed unweighted literals. The weight of an edge is the surplus of the constraint plus the sum of the weights of the clause's weighted variables (as these are likely to appear in the sum exactly once and so will have their coefficient rounded up thus increasing the slack).

- The weight of a node is the accumulated edge weights on the path from the start node.

- There are two nodes per variable. One node represents the length of the shortest odd path to the node. The other that of the shortest even path.

- The start node corresponds to an even length path to the start variable. This null path is considered even.

- The algorithm terminates when either:

1. The chosen node (most recently marked) has a weight exceeding 0.4 (indicating that any separating cut is no deeper than 0.3 , a failure), or has a length greater than 2 (a failure), or

2. The chosen node is the odd sense of the start variable. Thus, we have an odd path from the start node to itself (a cycle).

The odd cycle cuts can only be applied if we start with a large number of two variable clauses or after a significant number of variables have been fixed. Hence, these cuts tend to be applied only deep into the tree. We will investigate the effects of a more robust approach in a future paper. 
2.6. Branching. After generating cuts and applying bounds, we branch if there are still fractional variables in the LP solution.

The branching scheme we use is a modification of the Jeroslow-Wang [21] scheme.

We examine the probability of satisfying all the constraints if we randomly assign values to the remaining unfixed variables. For simplification, we assume that the probabilities of such an assignment satisfying each constraint are independent, and that each binary variable assignment has a probability of one half. We attempt to pick a variable whose assignment will have the greatest change in this probability.

For each fractional variable, we find a weight for the positive and negative sense as follows. We investigate all the clauses (original plus the resolvents) containing the given sense of the variable. We consider the effect of picking the opposite sense of the variable (making it harder to satisfy this constraint). By selecting the wrong choice we have turned the $k$ variable constraint into a $k-1$ variable constraint. The probability of satisfying this constraint is:

$$
1-\left(\frac{1}{2}\right)^{k-1}
$$

We wish to minimize the product of such terms (hence to minimize the sum of their logs). A similar approach is used for the opposite sense of the variable. We sum these two values and branch on the variable with the greatest sum. Such a variable will have a significant effect along either of the two branches and should, therefore, tend to generate a smaller branch and bound tree. For this variable, we first explore the more heavily weighted branch.

This approach differs from Jeroslow-Wang in that:

1. We give greater weight to the shorter clauses.

2. We consider the effect on both branches.

\section{Test Results}

In this section we compare our branch and cut code $(\mathrm{B}+\mathrm{C})$ to a MAX-SAT extension of the Davis-Putnam-Loveland (EDPL) algorithm [2]. Both are implemented in C. The branch and cut code uses the MINTO package of Savelsbergh et al. [27] (replacing default modules with those discussed in previous sections). All tests are executed on an IBM RS6000/390 with 128 Megabytes of memory. The abbreviation "MEM" in the tables indicates that a test terminated due to insufficient memory.

We tested both algorithms on a set of unweighted problems generated by the MWFF package of Selman [28]. Both MAX-2-SAT and MAX-3-SAT problems were examined. Various numbers of clauses were tried. This produced some problems that were satisfiable and others with several unsatisfiable clauses. For MAX-3SAT, we also tried different number of variables. The results were sorted on the number of unsatisfied clauses, as this gave a strong indication of the difficulty of the problem.

For MAX-2-SAT problems, the branch and cut code appears superior. EDPL only performs better on problems with a small number of clauses. Both algorithms take more CPU time as the number of clauses grow; however, the execution time of EDPL grew explosively. The branch and cut code tends to generate very small 


\begin{tabular}{|c|c|c|c|c|c|c|}
\hline \multirow{2}{*}{$\begin{array}{l}\text { problem } \\
\text { name }\end{array}$} & \multirow[t]{2}{*}{ clauses } & \multirow{2}{*}{$\begin{array}{l}\text { unsat. } \\
\text { clauses }\end{array}$} & \multicolumn{2}{|c|}{$\mathrm{B}+\mathrm{C}$} & \multicolumn{2}{|c|}{ EDPL } \\
\hline & & & $\mathrm{CPU}$ & nodes & $\mathrm{CPU}$ & backtracks \\
\hline p2180_5 & 180 & 0 & 0.84 & 1 & 0.1 & 1 \\
\hline p2180_8 & 180 & 1 & 5.20 & 1 & 0.7 & 2 \\
\hline p2180_2 & 180 & 2 & 5.44 & 1 & 0.7 & 10 \\
\hline p2180_3 & 180 & 2 & 5.25 & 1 & 0.7 & 40 \\
\hline p2180_6 & 180 & 2 & 5.03 & 1 & 0.7 & 47 \\
\hline p2180_9 & 180 & 2 & 5.39 & 1 & 0.7 & 34 \\
\hline p2180_1 & 180 & 3 & 5.09 & 1 & 0.7 & 162 \\
\hline p2180_7 & 180 & 4 & 5.41 & 1 & 0.8 & 964 \\
\hline p2180_4 & 180 & 4 & 5.43 & 1 & 0.9 & 1773 \\
\hline p2180_10 & 180 & 4 & 5.29 & 1 & 0.9 & 1933 \\
\hline p2200_8 & 200 & 4 & 5.23 & 1 & 0.8 & 1065 \\
\hline p2200_6 & 200 & 4 & 5.34 & 1 & 0.9 & 1304 \\
\hline p2200_3 & 200 & 4 & 5.43 & 1 & 0.9 & 2219 \\
\hline p2200_5 & 200 & 5 & 5.40 & 1 & 1.5 & 7982 \\
\hline p2200_1 & 200 & 5 & 5.62 & 1 & 2.3 & 12303 \\
\hline p2200_7 & 200 & 5 & 5.42 & 1 & 2.3 & 13549 \\
\hline p2200_2 & 200 & 6 & 5.57 & 1 & 4.2 & 32700 \\
\hline p2200_9 & 200 & 6 & 5.52 & 1 & 8.9 & 80824 \\
\hline p2200_10 & 200 & 6 & 5.95 & 3 & 8.2 & 73117 \\
\hline p2200_4 & 200 & 7 & 5.88 & 3 & 20.1 & 217362 \\
\hline
\end{tabular}

TABLE 1. Computational results for 100 variable MAX-2-SAT problems with a small number of clauses

trees while EDPL generates large ones. As a result, branch and cut performs dramatically better.

Our algorithm does not perform nearly so well for MAX-3-SAT problems. The search tree is generally smaller than that of EDPL. However, the evaluation at each node is much more expensive thus resulting in much greater execution times. EDPL's advantage diminishes with increasing numbers of unsatisfied clauses (for example the series of problems in Tables $5-7$ ); however, even here, EDPL performs better.

We also examine the Steiner "D" weighted tree problems [22] (see Table 13). Our current implementation of the primal heuristic is too primitive to handle these large variable problems well. So, for these problems, we run our code with the primal heuristic disabled. The EDPL code takes in excess of 12 hours on all of these problems. This is true even if the primal heuristic is disabled and the code is provided with the correct incumbent value. Our branch and cut code handles many of these problems with ease.

\section{Conclusions and Future Directions}

We compared two algorithms for solving MAX-SAT. Neither one was universally superior. The general trend was that branch and cut works best on MAX2-SAT and the Steiner "D" problems (where the average clause length is small). Trends suggest that branch and cut may also be better if the system contains a large 


\begin{tabular}{|l|r|r|r|r|r|r|}
\hline problem & clauses & unsat. & \multicolumn{2}{|c|}{ B+C } & \multicolumn{2}{|c|}{ EDPL } \\
\cline { 4 - 7 } name & & clauses & CPU & nodes & CPU & backtracks \\
\hline \hline p2220_5 & 220 & 4 & 5.73 & 1 & 0.8 & 1045 \\
\hline p2220_3 & 220 & 4 & 5.76 & 1 & 1.2 & 3699 \\
\hline p2220_9 & 220 & 4 & 5.83 & 1 & 1.0 & 2233 \\
\hline p2220_2 & 220 & 5 & 5.70 & 1 & 1.8 & 10039 \\
\hline p2220_7 & 220 & 6 & 5.81 & 1 & 5.7 & 48360 \\
\hline p2220_1 & 220 & 7 & 6.01 & 3 & 10.3 & 90586 \\
\hline p2220_10 & 220 & 7 & 6.23 & 3 & 9.8 & 91322 \\
\hline p2220_8 & 220 & 7 & 7.02 & 9 & 21.6 & 197734 \\
\hline p2220_4 & 220 & 8 & 6.19 & 3 & 17.4 & 172550 \\
\hline p2220_6 & 220 & 8 & 6.33 & 3 & 41.5 & 418421 \\
\hline \hline p2240_1 & 240 & 6 & 5.91 & 1 & 5.0 & 12402 \\
\hline p2240_7 & 240 & 7 & 6.85 & 3 & 26.6 & 242434 \\
\hline p2240_9 & 240 & 9 & 6.82 & 3 & 33.9 & 337982 \\
\hline p2240_10 & 240 & 9 & 6.96 & 3 & 84.2 & 817004 \\
\hline p2240_2 & 240 & 9 & 6.94 & 3 & 115.4 & 1150960 \\
\hline p2240_4 & 240 & 9 & 6.81 & 3 & 163.9 & 1767247 \\
\hline p2240_5 & 240 & 9 & 8.04 & 5 & 150.4 & 1634963 \\
\hline p2240_6 & 240 & 9 & 6.87 & 3 & 181.4 & 1775023 \\
\hline p2240_8 & 240 & 9 & 6.61 & 3 & 126.4 & 1357751 \\
\hline p2240_3 & 240 & 11 & 9.27 & 7 & 1205.4 & 13050216 \\
\hline
\end{tabular}

TABlE 2. Computational results for 100 variable MAX-2-SAT problems with a somewhat small number of clauses

number of unsatisfied clauses. EDPL appears to generally work best for MAX-3SAT. The EDPL code generates larger search trees, but spends much less time per node.

There are a number of possible directions to explore:

One possibility is some kind of hybrid algorithm, perhaps using a limited EDPL step for node fathoming and/or variable fixing. This should help reduce the tree size with a relatively low cost per node.

Another possibility is the addition of deeper cuts such as max-clique [3] inequalities.

A third possibility is a branch step that takes into account the slacks on the LP relaxation. It may also be worthwhile to investigate branching on several variables, perhaps reducing the total number of LP evaluations.

A final possibility is to reduce or eliminate cuts in the first few levels of the tree. Despite the addition of a large number of cuts here, the tree expanded in a binary fashion for several levels (see table 14). These cuts increase the size of the LP's and, therefore, the time to solve them. 


\begin{tabular}{|c|c|c|c|c|c|c|}
\hline \multirow{2}{*}{$\begin{array}{l}\text { problem } \\
\text { name }\end{array}$} & \multirow[t]{2}{*}{ clauses } & \multirow{2}{*}{$\begin{array}{l}\text { unsat. } \\
\text { clauses }\end{array}$} & \multicolumn{2}{|c|}{$\mathrm{B}+\mathrm{C}$} & \multicolumn{2}{|c|}{ EDPL } \\
\hline & & & $\mathrm{CPU}$ & nodes & $\mathrm{CPU}$ & backtracks \\
\hline$\overline{\text { p2260_10 }}$ & 260 & 6 & 6.49 & 1 & 4.1 & 29932 \\
\hline p2260_4 & 260 & 8 & 6.80 & 3 & 12.5 & 109971 \\
\hline p2260_7 & 260 & 8 & 6.10 & 1 & 36.4 & 340233 \\
\hline p2260_3 & 260 & 9 & 6.24 & 1 & 61.7 & 588508 \\
\hline p2260_2 & 260 & 9 & 6.54 & 3 & 66.2 & 631689 \\
\hline p2260_6 & 260 & 11 & 9.12 & 5 & 510.7 & 5214818 \\
\hline p2260_8 & 260 & 11 & 9.33 & 5 & 939.1 & 9722169 \\
\hline p2260_1 & 260 & 11 & 11.37 & 9 & 1543.1 & 15149775 \\
\hline p2260_9 & 260 & 12 & 12.36 & 9 & 2556.3 & 26165444 \\
\hline p2260_5 & 260 & 12 & 11.38 & 7 & 4293.8 & 46674569 \\
\hline p2280_10 & 280 & 10 & 7.49 & 3 & 227.2 & 2118261 \\
\hline p2280_5 & 280 & 11 & 7.98 & 3 & 1398.0 & 14876069 \\
\hline p2280_8 & 280 & 11 & 8.50 & 5 & 495.6 & 4770966 \\
\hline p2280_9 & 280 & 11 & 8.24 & 3 & 407.3 & 3853794 \\
\hline p2280_7 & 280 & 12 & 8.87 & 5 & 3437.0 & 35347297 \\
\hline p2280_1 & 280 & 13 & 12.29 & 7 & 2738.0 & 28997281 \\
\hline p2280_3 & 280 & 13 & 13.34 & 11 & 3151.6 & 32091970 \\
\hline p2280_6 & 280 & 14 & 19.82 & 15 & 29111.1 & 313402437 \\
\hline p2280_2 & 280 & 15 & 19.53 & 13 & 32085.0 & 332713465 \\
\hline p2280_4 & 280 & 15 & 20.81 & 15 & 45268.2 & 503462478 \\
\hline
\end{tabular}

TABLE 3. Computational results for 100 variable MAX-2-SAT problems with a medium number of clauses 


\begin{tabular}{|c|c|c|c|c|c|c|}
\hline \multirow{2}{*}{$\begin{array}{l}\text { problem } \\
\text { name }\end{array}$} & \multirow[t]{2}{*}{ clauses } & \multirow{2}{*}{$\begin{array}{l}\text { unsat. } \\
\text { clauses }\end{array}$} & \multicolumn{2}{|c|}{$\mathrm{B}+\mathrm{C}$} & \multicolumn{2}{|c|}{ EDPL } \\
\hline & & & $\mathrm{CPU}$ & nodes & $\mathrm{CPU}$ & backtracks \\
\hline $\bar{p} 2300 \_2$ & 300 & 13 & 8.27 & 3 & 2229.1 & 21334438 \\
\hline p2300_3 & 300 & 13 & 8.22 & 3 & 2497.9 & 23764177 \\
\hline $\mathrm{p} 2300 \_4$ & 300 & 14 & 10.96 & 5 & 4618.5 & 47629271 \\
\hline p2300_1 & 300 & 15 & 20.04 & 11 & 10564.3 & 104553113 \\
\hline p2300_5 & 300 & 15 & 17.63 & 11 & 29738.0 & 309999407 \\
\hline p2300_9 & 300 & 15 & 18.61 & 11 & 8746.0 & 85235320 \\
\hline p2300_10 & 300 & 15 & 19.16 & 11 & 12693.1 & 125325067 \\
\hline p2300_6 & 300 & 17 & 64.57 & 35 & 69325.3 & 704246333 \\
\hline p2300_8 & 300 & 17 & 37.23 & 19 & Not Run & Not Run \\
\hline $\mathrm{p} 2300 \_7$ & 300 & 20 & 92.97 & 45 & Not Run & Not Run \\
\hline p2400_3 & 400 & 25 & 80.08 & 27 & Not Run & Not Run \\
\hline $\mathrm{p} 2400 \_7$ & 400 & 26 & 102.35 & 29 & Not Run & Not Run \\
\hline p2400_6 & 400 & 27 & 117.51 & 35 & Not Run & Not Run \\
\hline p2400_2 & 400 & 28 & 83.44 & 27 & Not Run & Not Run \\
\hline $\mathrm{p} 2400 \_4$ & 400 & 28 & 115.30 & 29 & Not Run & Not Run \\
\hline p2400_1 & 400 & 29 & 122.42 & 37 & Not Run & Not Run \\
\hline p2400_5 & 400 & 29 & 226.72 & 57 & Not Run & Not Run \\
\hline p2400_10 & 400 & 30 & 565.93 & 135 & Not Run & Not Run \\
\hline p2400_8 & 400 & 33 & 518.31 & 103 & Not Run & Not Run \\
\hline p2400_9 & 400 & 34 & 1518.91 & 281 & Not Run & Not Run \\
\hline
\end{tabular}

TABLE 4. Computational results for 100 variable MAX-2-SAT problems with a large number of clauses

\begin{tabular}{|l|r|r|r|r|r|r|}
\hline \multirow{2}{*}{$\begin{array}{l}\text { problem } \\
\text { name }\end{array}$} & \multirow{2}{*}{ clauses } & unsat. & \multicolumn{2}{|c|}{ B+C } & \multicolumn{2}{|c|}{ EDPL } \\
\cline { 4 - 7 } & & clauses & CPU & nodes & CPU & backtracks \\
\hline \hline test215_1 & 215 & 0 & 0.81 & 1 & 0.1 & 1 \\
\hline test215_3 & 215 & 1 & 5.29 & 15 & 0.5 & 23 \\
\hline test215_2 & 215 & 1 & 5.76 & 23 & 0.5 & 25 \\
\hline test215_5 & 215 & 1 & 6.52 & 33 & 0.5 & 31 \\
\hline test215_4 & 215 & 2 & 34.47 & 353 & 0.6 & 562 \\
\hline \hline test250_3 & 250 & 0 & 2.63 & 1 & 0.2 & 1 \\
\hline test250_1 & 250 & 2 & 17.27 & 89 & 0.6 & 314 \\
\hline test250_4 & 250 & 2 & 21.52 & 123 & 0.6 & 306 \\
\hline test250_5 & 250 & 2 & 28.53 & 177 & 0.6 & 362 \\
\hline test250_2 & 250 & 4 & 253.76 & 1467 & 3.2 & 20001 \\
\hline
\end{tabular}

TABLE 5. Computational results for 50 variable MAX-3-SAT problems with a small number of clauses 


\begin{tabular}{|l|r|r|r|r|r|r|}
\hline problem & clauses & unsat. & \multicolumn{2}{|c|}{ B+C } & \multicolumn{2}{|c|}{ EDPL } \\
\cline { 4 - 7 } name & & clauses & CPU & nodes & CPU & backtracks \\
\hline \hline test300_3 & 300 & 4 & 78.80 & 275 & 2.1 & 10116 \\
\hline test300_1 & 300 & 4 & 147.10 & 577 & 2.3 & 11073 \\
\hline test300_2 & 300 & 5 & 249.48 & 891 & 5.9 & 42151 \\
\hline test300_5 & 300 & 5 & 325.48 & 1107 & 8.4 & 59216 \\
\hline test300_4 & 300 & 6 & 493.78 & 1583 & 16.2 & 130802 \\
\hline \hline test350_4 & 350 & 5 & 168.53 & 417 & 4.8 & 28409 \\
\hline test350_2 & 350 & 6 & 460.75 & 1195 & 15.1 & 112539 \\
\hline test350_1 & 350 & 8 & 1718.31 & 3867 & 103.3 & 915731 \\
\hline test350_3 & 350 & 8 & 1877.87 & 4363 & 97.8 & 864778 \\
\hline test350_5 & 350 & 8 & 2369.56 & 5659 & 118.0 & 1048876 \\
\hline \hline test400_3 & 400 & 8 & 758.43 & 1349 & 62.8 & 482523 \\
\hline test400_2 & 400 & 8 & 1163.13 & 2101 & 68.9 & 521495 \\
\hline test400_5 & 400 & 8 & 1020.13 & 1895 & 76.1 & 594532 \\
\hline test400_4 & 400 & 11 & 4249.03 & 6341 & 519.8 & 4541184 \\
\hline test400_1 & 400 & 11 & 6501.42 & 9701 & 690.1 & 6196183 \\
\hline \hline
\end{tabular}

TABLE 6. Computational results for 50 variable MAX-3-SAT problems with a medium number of clauses

\begin{tabular}{|l|r|r|r|r|r|r|}
\hline problem & \multirow{2}{*}{ clauses } & unsat. & \multicolumn{2}{|c|}{ B+C } & \multicolumn{2}{|c|}{ EDPL } \\
\cline { 4 - 7 } name & & clauses & CPU & nodes & CPU & backtracks \\
\hline \hline test450_4 & 450 & 10 & 1522.54 & 2139 & 162.6 & 1237498 \\
\hline test450_5 & 450 & 11 & 2326.24 & 3003 & 357.9 & 2817502 \\
\hline test450_3 & 450 & 11 & 3676.28 & 4549 & 533.7 & 4268781 \\
\hline test450_1 & 450 & 12 & 5480.85 & 6197 & 868.9 & 7129533 \\
\hline test450_2 & 450 & 14 & 13440.50 & 14077 & 2752.4 & 24408217 \\
\hline \hline test500_1 & 500 & 15 & 9324.21 & 7505 & 2465.1 & 19442201 \\
\hline test500_3 & 500 & 16 & 17271.87 & 13443 & 4081.6 & 32959363 \\
\hline test500_4 & 500 & 16 & 17460.65 & 13387 & 3923.8 & 31096996 \\
\hline test500_5 & 500 & 19 & MEM & MEM & 15832.7 & 136515702 \\
\hline
\end{tabular}

TABLE 7. Computational results for 50 variable MAX-3-SAT problems with a large number of clauses 


\begin{tabular}{|l|r|r|r|r|r|r|}
\hline \multirow{2}{*}{$\begin{array}{l}\text { problem } \\
\text { name }\end{array}$} & clauses & unsat. & \multicolumn{2}{|c|}{ B+C } & \multicolumn{2}{|c|}{ EDPL } \\
\cline { 4 - 7 } clauses & CPU & nodes & CPU & backtracks \\
\hline \hline s323_1 & 323 & 0 & 1.37 & 1 & 0.2 & 1 \\
\hline s323_2 & 323 & 0 & 2.02 & 1 & 0.1 & 1 \\
\hline s323_3 & 323 & 0 & 1.75 & 1 & 0.1 & 1 \\
\hline s323_4 & 323 & 0 & 1.01 & 1 & 0.1 & 1 \\
\hline s323_5 & 323 & 1 & 18.39 & 77 & 0.8 & 94 \\
\hline \hline s350_1 & 350 & 1 & 15.80 & 45 & 0.9 & 85 \\
\hline s350_2 & 350 & 1 & 20.68 & 79 & 0.9 & 76 \\
\hline s350_3 & 350 & 1 & 21.09 & 89 & 0.9 & 77 \\
\hline s350_4 & 350 & 1 & 21.05 & 87 & 0.9 & 73 \\
\hline s350_5 & 350 & 2 & 143.99 & 749 & 1.3 & 1176 \\
\hline \hline s375_1 & 375 & 0 & 2.41 & 1 & 0.1 & 1 \\
\hline s375_2 & 375 & 2 & 139.37 & 557 & 1.5 & 1527 \\
\hline s375_3 & 375 & 2 & 199.87 & 907 & 1.5 & 1649 \\
\hline s375_5 & 375 & 3 & 437.32 & 1477 & 4.0 & 11649 \\
\hline s375_4 & 375 & 3 & 537.77 & 1917 & 4.7 & 14669 \\
\hline \multicolumn{3}{|c|}{ TABLE } \\
problems with a small number of clauses & MAX-3-SAT \\
\hline
\end{tabular}

\begin{tabular}{|l|r|r|r|r|r|r|}
\hline problem & clauses & unsat. & \multicolumn{2}{|c|}{ B+C } & \multicolumn{2}{|c|}{ EDPL } \\
\cline { 4 - 7 } name & & clauses & CPU & nodes & CPU & backtracks \\
\hline \hline s400_3 & 400 & 2 & 190.70 & 733 & 1.7 & 1894 \\
\hline s400_5 & 400 & 3 & 653.70 & 2049 & 4.1 & 11460 \\
\hline s400_2 & 400 & 4 & 1300.32 & 3463 & 20.3 & 90478 \\
\hline s400_4 & 400 & 4 & 1874.79 & 5369 & 25.9 & 118064 \\
\hline s400_1 & 400 & 5 & 4276.55 & 10353 & 104.1 & 554165 \\
\hline \hline s425_1 & 425 & 4 & 1689.55 & 3869 & 20.4 & 83776 \\
\hline s425_3 & 425 & 4 & 1841.67 & 4439 & 21.8 & 88537 \\
\hline s425_4 & 425 & 5 & 3378.52 & 7815 & 84.6 & 442233 \\
\hline s425_2 & 425 & 5 & 5214.78 & 11643 & 110.0 & 559420 \\
\hline s425_5 & 425 & 6 & MEM & MEM & 478.5 & 2799709 \\
\hline \hline s450_4 & 450 & 5 & 3761.84 & 7149 & 97.9 & 481736 \\
\hline s450_5 & 450 & 6 & 7208.57 & 13225 & 295.9 & 1603476 \\
\hline s450_1 & 450 & 6 & MEM & MEM & 466.4 & 2619212 \\
\hline s450_2 & 450 & 7 & MEM & MEM & 1639.2 & 10218689 \\
\hline s450_3 & 450 & 7 & MEM & MEM & 1212.7 & 7362249 \\
\hline
\end{tabular}




\begin{tabular}{|l|r|r|r|r|r|r|}
\hline problem & \multirow{2}{*}{ clauses } & \multirow{2}{*}{$\begin{array}{c}\text { unsat. } \\
\text { name }\end{array}$} & & \multicolumn{2}{|c|}{ B+C } & \multicolumn{2}{|c|}{ EDPL } \\
\cline { 4 - 7 } & & clauses & CPU & nodes & CPU & backtracks \\
\hline \hline s475_2 & 475 & 6 & 6105.25 & 9767 & 345.5 & 1844920 \\
\hline s475_3 & 475 & 6 & 6258.74 & 9765 & 282.3 & 1500066 \\
\hline s475_5 & 475 & 7 & 7668.66 & 10287 & 678.2 & 3741527 \\
\hline s475_1 & 475 & 7 & 12541.21 & 18867 & 856.5 & 4841953 \\
\hline s475_4 & 475 & 8 & MEM & MEM & 2651.3 & 15909584 \\
\hline \hline s500_2 & 500 & 7 & 9331.30 & 12099 & 758.0 & 4205882 \\
\hline s500_4 & 500 & 7 & MEM & MEM & 985.3 & 5693206 \\
\hline s500_3 & 500 & 8 & MEM & MEM & 2652.9 & 15799725 \\
\hline
\end{tabular}

TABLE 10. Computational results for 75 variable MAX-3-SAT problems with a large number of clauses

\begin{tabular}{|c|c|c|c|c|c|c|}
\hline \multirow{2}{*}{$\begin{array}{l}\text { problem } \\
\text { name }\end{array}$} & \multirow[t]{2}{*}{ clauses } & \multirow{2}{*}{$\begin{array}{l}\text { unsat. } \\
\text { clauses }\end{array}$} & \multicolumn{2}{|c|}{$\mathrm{B}+\mathrm{C}$} & \multicolumn{2}{|c|}{ EDPL } \\
\hline & & & CPU & nodes & CPU & backtracks \\
\hline o430_4 & 430 & 0 & 1.66 & 1 & 0.1 & 1 \\
\hline o430_2 & 430 & 0 & 3.67 & 1 & 0.1 & 1 \\
\hline O430_3 & 430 & 0 & 1.75 & 1 & 0.2 & 1 \\
\hline o430_1 & 430 & 1 & 120.85 & 545 & 1.4 & 320 \\
\hline o430_5 & 430 & 2 & 1833.07 & 7991 & 5.2 & 8834 \\
\hline $0450 \_5$ & 450 & 0 & 1.50 & 1 & 0.1 & 1 \\
\hline o450_4 & 450 & 1 & 65.81 & 247 & 1.3 & 199 \\
\hline O450_3 & 450 & 1 & 87.50 & 347 & 1.4 & 312 \\
\hline o450_1 & 450 & 2 & 988.54 & 3589 & 3.5 & 5221 \\
\hline o450_2 & 450 & 2 & 1336.35 & 4667 & 3.9 & 5712 \\
\hline o475_3 & 475 & 1 & 72.03 & 251 & 4.1 & 6168 \\
\hline o475_4 & 475 & 1 & 72.81 & 253 & 1.4 & 230 \\
\hline $0475 \_5$ & 475 & 1 & 65.66 & 213 & 1.4 & 281 \\
\hline o475_1 & 475 & 2 & 593.13 & 2059 & 3.2 & 4212 \\
\hline o475_2 & 475 & 2 & 1028.67 & 3347 & 3.3 & 4265 \\
\hline o500_1 & 500 & 0 & 2.47 & 1 & 0.2 & 1 \\
\hline O500_3 & 500 & 2 & 1068.16 & 3389 & 2.9 & 3345 \\
\hline 0500_2 & 500 & 3 & 4795.02 & 12683 & 31.3 & 87816 \\
\hline $0500 \_4$ & 500 & 4 & MEM & MEM & 162.5 & 572489 \\
\hline $0500 \_5$ & 500 & 4 & $\overline{\mathrm{MEM}}$ & MEM & 203.1 & 735535 \\
\hline
\end{tabular}

TABLE 11. Computational results for 100 variable MAX-3-SAT problems with a small number of clauses 


\begin{tabular}{|l|r|r|r|r|r|r|}
\hline problem & clauses & unsat. & \multicolumn{2}{|c|}{ B+C } & \multicolumn{2}{|c|}{ EDPL } \\
\cline { 4 - 7 } name & & clauses & CPU & nodes & CPU & backtracks \\
\hline \hline o525_1 & 525 & 2 & 493.49 & 1287 & 2.3 & 1910 \\
\hline o525_4 & 525 & 3 & 3640.80 & 8073 & 21.3 & 54483 \\
\hline o525_3 & 525 & 3 & 5379.72 & 12301 & 29.0 & 74311 \\
\hline o525_5 & 525 & 3 & 5596.78 & 11893 & 25.7 & 66390 \\
\hline o525_2 & 525 & 5 & MEM & MEM & 1122.6 & 4525046 \\
\hline \hline o550_2 & 550 & 3 & 2162.29 & 3969 & 11.2 & 24487 \\
\hline o550_1 & 550 & 3 & 3746.04 & 7367 & 30.0 & 78768 \\
\hline o550_5 & 550 & 4 & 16601.80 & 28423 & 147.9 & 479767 \\
\hline 0550_3 & 550 & 5 & MEM & MEM & 837.4 & 3304456 \\
\hline 0550_4 & 550 & 6 & MEM & MEM & 7526.6 & 33281390 \\
\hline \hline 0575_5 & 575 & 4 & 12477.14 & 19139 & 151.9 & 491192 \\
\hline o575_3 & 575 & 5 & MEM & MEM & 602.2 & 2191647 \\
\hline o575_1 & 575 & 6 & MEM & MEM & 4370.9 & 18334787 \\
\hline 0575_2 & 575 & 7 & MEM & MEM & 10488.5 & 47346157 \\
\hline
\end{tabular}

TABLE 12. Computational results for 100 variable MAX-3-SAT problems with a large number of clauses

\begin{tabular}{|l|r|r|r|r|r|r|}
\hline problem & variables & clauses & ave clause & optimal & \multicolumn{2}{|c|}{ B+C } \\
\cline { 6 - 7 } name & & & 1 length & value & CPU & nodes \\
\hline \hline steind2 & 1295 & 1765 & 1.31 & 220 & 4423.23 & 7599 \\
\hline steind3 & 1416 & 1885 & 1.25 & 1646 & 3.63 & 1 \\
\hline steind4 & 1499 & 2074 & 1.28 & 2044 & 5.08 & 1 \\
\hline steind5 & 1749 & 2646 & 1.34 & 3419 & 14.47 & 1 \\
\hline steind7 & 2045 & 2325 & 1.15 & 103 & 6.53 & 7 \\
\hline steind8 & 2166 & 2544 & 1.15 & 1180 & 3.46 & 1 \\
\hline steind9 & 2249 & 2797 & 1.20 & 1585 & 4.84 & 1 \\
\hline steind10 & 2499 & 3261 & 1.23 & 2219 & 12.68 & 1 \\
\hline steind11 & 5120 & 5882 & 1.17 & 29 & 2374.98 & 523 \\
\hline steind12 & 5009 & 5039 & 1.01 & 42 & 2.78 & 1 \\
\hline steind13 & 5166 & 5499 & 1.06 & 544 & 4.76 & 1 \\
\hline steind14 & 5249 & 5709 & 1.08 & 740 & 6.33 & 1 \\
\hline steind15 & 5499 & 6202 & 1.11 & 1193 & 13.49 & 1 \\
\hline steind16 & 25032 & 25133 & 1.01 & 13 & 208.37 & 4 \\
\hline steind18 & 25166 & 25412 & 1.01 & 262 & 13.32 & 1 \\
\hline steind19 & 25249 & 25585 & 1.01 & 359 & 15.59 & 1 \\
\hline steind20 & 25499 & 26041 & 1.02 & 558 & 21.45 & 1 \\
\hline
\end{tabular}

TABle 13. Computational results for Steiner "D" tree problems 


\begin{tabular}{|l|r|r|r|}
\hline depth & nodes & $\begin{array}{r}\text { resolution } \\
\text { cuts/node }\end{array}$ & $\begin{array}{r}\text { odd cycle } \\
\text { cuts/node }\end{array}$ \\
\hline \hline 0 & 1 & 0.000 & 0.000 \\
\hline 1 & 2 & 1.500 & 0.000 \\
\hline 2 & 4 & 6.250 & 0.500 \\
\hline 3 & 8 & 19.000 & 1.250 \\
\hline 4 & 16 & 12.875 & 2.625 \\
\hline 5 & 32 & 13.344 & 3.875 \\
\hline 6 & 64 & 11.594 & 6.719 \\
\hline 7 & 128 & 8.438 & 7.781 \\
\hline 8 & 256 & 7.516 & 8.609 \\
\hline 9 & 512 & 7.227 & 9.416 \\
\hline 10 & 971 & 7.270 & 9.747 \\
\hline 11 & 1461 & 7.600 & 9.721 \\
\hline 12 & 1674 & 7.224 & 9.648 \\
\hline 13 & 1428 & 7.429 & 9.148 \\
\hline 14 & 991 & 7.272 & 8.954 \\
\hline 15 & 593 & 6.390 & 8.155 \\
\hline 16 & 316 & 5.551 & 6.965 \\
\hline 17 & 180 & 6.172 & 5.989 \\
\hline 18 & 102 & 7.686 & 5.912 \\
\hline 19 & 67 & 6.060 & 5.776 \\
\hline 20 & 40 & 4.775 & 4.525 \\
\hline 21 & 27 & 4.704 & 3.111 \\
\hline 22 & 10 & 3.700 & 2.300 \\
\hline 23 & 4 & 2.750 & 1.750 \\
\hline 24 & 1 & 0.000 & 0.000 \\
\hline & $1 \times 18$ & 614 & \\
\hline
\end{tabular}

TABLE 14. Cuts per node in the branch and cut tree of problem test500_4 in table 7 


\section{References}

[1] C. E. Blair, R. G. Jeroslow, and J. K. Lowe. Some results and experiments in programming techniques for propositional logic. Computers and Operations Research, 13(5):633-645, 1986.

[2] B. Borchers and J. Furman. A two-phase exact algorithm for MAX-SAT and weighted MAXSAT problems. Technical report, Mathematics Department, New Mexico Tech, Socorro, NM 87801, October 1995.

[3] J. Cheriyan, W. H. Cunningham, L. Tunçel, and Y. Wang. A linear programming and rounding approach to max 2-sat. Technical report, Department of Combinatorics and Optimization, University of Waterloo, Waterloo, Canada N2L 3G1, 1996. To appear in: Cliques, Coloring, and Satisfiability: Second DIMACS Implementation Challenge, D. S. Johnson and M. A. Trick (eds), DIMACS Series in Discrete Mathematics and Theoretical Computer Science, AMS, 1995.

[4] M. Davis and H. Putnam. A computing procedure for quantification theory. J. Assoc. Comput. Mach., 7:201-215, 1960 .

[5] Michael R. Garey and David S. Johnson. Computers and Intractability: A Guide to the Theory of NP-Completeness. W. H. Freeman and Company, 1979.

[6] Michel X. Goemans and David P. Williamson. Improved Approximation Algorithms for Maximum Cut and Satisfiability Problems Using Semidefinite Programming. J. Assoc. Comput. Mach., 42(6):1115-1145, 1995.

[7] M. Grötschel and O. Holland. Solution of large-scale travelling salesman problems. Mathematical Programming, 51(2):141-202, 1991.

[8] M. Grötschel, M. Jünger, and G. Reinelt. A cutting plane algorithm for the linear ordering problem. Operations Research, 32:1195-1220, 1984.

[9] J. Gu. Parallel Algorithms and Architectures for Very Fast AI Search. PhD thesis, University of Utah, 1989.

[10] J. Gu. Efficient local search for very large-scale satisfiability problem. SIGART Bulletin, 3(1):8-12, January 1992, ACM Press.

[11] J. Gu. Local search for satisfiability (SAT) problem. IEEE Trans. on Systems, Man, and Cybernetics, 23(4):1108-1129, Jul. 1993, and 24(4):709, Apr. 1994.

[12] J. Gu. Global optimization for satisfiability (SAT) problem. IEEE Trans. on Knowledge and Data Engineering, 6(3):361-381, Jun. 1994, and 7(1):192, Feb. 1995.

[13] J. Gu. Parallel algorithms for satisfiability (SAT) problem. DIMACS Series on Discrete Mathematics and Theoretical Computer Science - Parallel Processing on Discrete Optimization Problems, 22:105-161, Jul. American Mathematical Society, 1995.

[14] J. Gu, P.W. Purdom, J. Franco, and B.W. Wah. Algorithms for satisfiability (SAT) problem: A survey. DIMACS Volume Series on Discrete Mathematics and Theoretical Computer Science: The Satisfability (SAT) Problem, American Mathematical Society, 1996.

[15] P. Hansen and B. Jaumard. Algorithms for the maximum satisfiability problem. Computing, 44:279-303, 1990.

[16] F. Harche and G. L. Thompson. The column subtraction algorithm: An exact method for solving weighted set covering, packing and partitioning problems. Computers and Operations Research, 21:689-705, 1994.

[17] K. L. Hoffman and M. Padberg. Solving airline crew scheduling problems by branch-and-cut. Management Science, 39(6):657-682, 1993.

[18] J. N. Hooker. Resolution vs. cutting plane solution of inference problems: some computational experience. Operations Research Letters, 7(1):1-7, 1988.

[19] J. N. Hooker. Resolution and the integrality of satisfiability problems. Technical report, Carnegie Mellon University, 1995. To appear in: Mathematical Programming.

[20] J. N. Hooker and C. Fedjki. Branch-and-cut solution of inference problems in propositional logic. Annals of Mathematics and Artificial Intelligence, 1:123-139, 1990.

[21] R. G. Jeroslow and J. Wang. Solving propositional satisfiability problems. Annals of Mathematics and $A I, 1: 167-187,1990$.

[22] Y. Jiang, H. Kautz, and B. Selman. Solving problems with hard and soft constraints using a stochastic algorithm for MAX-SAT. Technical report, AT\&T Bell Laboratories, Murray Hill, NJ, 07974, 1995.

[23] D. Loveland. Automated Theorem-Proving: A Logical Basis. North-Holland, New York, 1978.

[24] M. W. Padberg and G. Rinaldi. A branch-and-cut algorithm for the resolution of large-scale symmetric traveling salesman problems. SIAM Review, 33(1):60-100, 1991. 
[25] Mauricio G. C. Resende and Thomas A. Feo. A grasp for satisfiability. Technical report, Department of Combinatorics and Optimization, University of Waterloo, 1995. To appear in: Cliques, Coloring, and Satisfiability: Second DIMACS Implementation Challenge, D. S. Johnson and M. A. Trick (eds), DIMACS Series in Discrete Mathematics and Theoretical Computer Science, AMS, 1995.

[26] J. A. Robinson. A machine-oriented logic based on the resolution principle. Journal of the ACM, 12:23-41, 1965.

[27] M. W. P. Savelsbergh, G. C. Sigismondi, and G. L. Nemhauser. MINTO: a Mixed INTeger Optimizer. Technical Report Memorandum COSOR 91-18, Eindhoven University of Technology, 1991.

[28] B. Selman. Mwff: Program for generating random max k-sat instances. available from DIMACS.

[29] B. Selman and H. A. Kautz. An empirical study of greedy local search for satisfiability testing. In Proceedings of the Eleventh National Conference on Artificial Intelligence (AAAI-92), San Jose, CA, 1993.

[30] B. Selman, H. Levesque, and D. Mitchell. A new method for solving hard satisfiability problems. In Proceedings of the Tenth National Conference on Artificial Intelligence (AAAI-92), San Jose, CA, pages 440-446, July 1992.

Mathematics Department, Rensselaer Polytechnic Institute, Troy, NY 12180

E-mail address: joys@rpi.edu

Mathematics Department, Rensselaer Polytechnic Institute, Troy, NY 12180

E-mail address: mitchj@rpi.edu

Mathematics Department, New Mexico Tech, Socorro, NM 87801

E-mail address: borchers@nmt.edu 